\title{
A strategic approach for preparation of oxide nanomaterials
}

\author{
K J RAO*, KRISHNAMURTHY MAHESH ${ }^{\dagger}$ and SUNDEEP KUMAR \\ Solid State and Structural Chemistry Unit, Indian Institute of Science, Bangalore 560 012, India
}

MS received 30 November 2004

\begin{abstract}
A microwave assisted solvothermal method is described for rapid preparation of nano-oxides. This method is based on exploiting differential dielectric constants to induce preferred heating and decomposition of the oxide precursors in the presence of suitable capping agents. This strategic approach has been used to prepare nanopowders of $\mathrm{MgO}, \mathrm{NiO}, \mathrm{ZnO}, \mathrm{Al}_{2} \mathrm{O}_{3}, \mathrm{Fe}_{2} \mathrm{O}_{3}$ and $\mathrm{ZrO}_{2}$.
\end{abstract}

Keywords. Microwave synthesis; nanomaterials; oxide powders.

\section{Introduction}

Nanomaterials research has witnessed an exponential growth during the last decade (Edelstein and Cammarata 1998; Esaki 1999; Ding et al 2003; He et al 2003; Pang et al 2003; Sankaranarayanan and Sreekumar 2003; Yin et al 2004). In the nanometric range, metallic and small band gap semiconducting materials exhibit fascinating quantum phenomena (Esaki 1999; Savchuk et al 2003). Large band gap materials such as oxides stabilize in their high temperature phases and exhibit enhanced surface phenomena like catalysis and reduced reaction barriers for solid-state reactions (Agrafiotis et al 2000; Kaskel et al 2004; Anderson et al 2004). Indeed, in the nanometric range, materials may be expected to behave quite differently from both molecular and bulk states since the ratio of the number of surface atoms to the number of bulk atoms is quite high. As a consequence of this increased ratio the number of valence unsatiated atoms in the nanoparticle becomes significant. There is thus a curiosity to understand the behaviour of materials in the nano length scale and if possible to exploit the new properties exhibited by materials purely as a consequence of the smallness of size. Nano oxide materials have found wide ranging applications particularly as catalysts and as starting materials for making advanced structural ceramics. During sintering and shaping of oxidic materials for practical applications, use of nano sized particles as starting materials can be of great advantage because of the availability of large surface areas of the nanoparticles. It may be noted that surface areas of particles for a given quantity of the material scales as $1 /\langle d\rangle$. Where $\langle d\rangle$ is the average diameter (linear dimension) of the particles.

\footnotetext{
*Author for correspondence (kjrao@ sscu.iisc.ernet.in) ${ }^{\dagger}$ Under graduate Summer Research Fellow of the Indian Academy of Sciences from IIT Madras.
}

Therefore, several methods have been developed for the preparation of nanomaterials (Edelstein and Cammarata 1998; Lee and Kim 2004; Pitisescu et al 2004). We have reported earlier (Rao et al 1998; Shen et al 2003; Lee and Choi 2004) from this laboratory that metal organics subjected to microwave irradiation decompose and form oxides often in the form of nanoparticles when subjected to microwave irradiation. Nanometric products also result when a gaseous reactant is used in microwave synthesis as observed in the preparation of aluminium nitride by carbothermal reduction cum nitridation method (Panneerselvam 2002). Recently, a solvothermal microwave method has been described for the preparation of nanometric metal particles (Komarneni 2003). However, no general strategy has yet been described for the preparation of nanomaterials based on the use of microwaves. In this communication we report a novel microwave method for the preparation of oxide nanoparticles. The method is based on a strategy that is of very general applicability. Preparation of a few oxides is described which demonstrates the validity of the strategy. Of these oxides chosen, $\mathrm{MgO}, \mathrm{NiO}, \mathrm{ZnO}$, $\mathrm{Al}_{2} \mathrm{O}_{3}, \mathrm{Fe}_{2} \mathrm{O}_{3}$ and $\mathrm{ZrO}_{2}$ have been prepared in nanometric form by other methods and have been reported in the literature. We suggest that the method described below can be extended for the preparation of nano materials other than oxides.

\section{Basis of the microwave strategy}

The approach is based on the following observations. When materials are irradiated with microwaves they absorb energy from the microwave field. The power absorbed, $P$, is given by

$$
P=2 \pi f \varepsilon_{0} \varepsilon^{\prime} \tan \delta\left|E_{\mathrm{i}}\right|^{2} .
$$

The frequency, $f$ and the amplitude $\left|E_{\mathrm{i}}\right|$ of the microwaves are instrumental parameters (controllable) while the dielectric constant, $\varepsilon^{\prime}$, and the loss tangent, $\tan \delta$, are 
material parameters $\left(\varepsilon_{0}\right.$ is the permittivity of the free space). Therefore, in a given instrumental set up $\left(\left|\Delta E_{\mathrm{i}}\right|\right.$ and $f$ held constants), $P$ is determined by $\varepsilon^{\prime}$ and $\tan \delta$. $\varepsilon^{\prime}$ is generally high for materials containing molecules or complex ions of high dipole moment and therefore, high molar polarization. Therefore, when a mixture of two materials, $\mathrm{A}$ and $\mathrm{B}$ of different dielectric constants, $\varepsilon_{\mathrm{A}}^{\prime}$ and $\varepsilon_{\mathrm{B}}^{\prime}$, is exposed to microwave field, the material with higher values of $\varepsilon^{\prime}$ absorbs energy preferentially and gets heated rapidly compared to the other. For the microwave decomposition of a material in a mixture the differential absorption of microwave energy can be used to advantage. A precursor material can be chosen such that it has a high value of $\varepsilon^{\prime}$ and decomposes by preferential microwave absorption to yield the desired oxide. Thus, if a metal organic or an inorganic complex salt, of reasonably high value of dielectric constant that yields the desired oxide upon decomposition is dissolved in a suitable liquid medium of low dielectric constant and irradiated with microwaves, we can expect the formation of the metal oxide. The oxide generally forms as a suspension in the chosen liquid. It would of course be better to choose a precursor, which has high values of both $\varepsilon^{\prime}$ and $\tan \delta$, but $\tan \delta$ values are often not readily available and are also highly temperature dependent. This may result in a disadvantage occasionally if $\tan \delta$ decreases with temperature (as in a post transition region) in the region where $\varepsilon^{\prime}$ increases. We have not investigated these aspects fully in this communication. The liquid chosen should be one, which does not decompose before its normal boiling temperature. Also the resulting oxide should not be soluble in it. The insoluble oxide product, however, should be prevented from growing into large particles. This is accomplished by the addition of a suitable capping agent to the initial solution. It is also necessary to control the microwave power so that the solvent does not boil violently.

\section{Experimental}

Six different oxides were prepared using the above strategy as described below. The starting materials used for their preparation are given in table 1 along with their dielectric constant values. These dielectric constant values were determined by measuring $\varepsilon^{\prime}(\omega)$ over a wide fre- quency range and extrapolating $\varepsilon^{\prime}(\omega)$ to $\omega=0$. Measurements were done on HP4192A Impedance Analyser. A special cell was designed to measure $\varepsilon^{\prime}(\omega)$ of liquid precursors. Generally a precursor concentration of $5 \mathrm{~g} / 100 \mathrm{ml}$ of solvent was employed. $0 \cdot 2 \mathrm{~g}$ (per $100 \mathrm{ml}$ of solvent) of poly vinyl pyrrolidone (PVP) was added as a capping agent. A homogeneous solution so obtained was kept in a round bottom flask fitted with a water-cooled refluxing system. A domestic (LG Scientific MS283MC $800 \mathrm{~W}$ ) microwave oven operating at $2.45 \mathrm{GHz}$ and with a sixstage variable power was slightly modified to accommodate the round bottomed flask with the attached refluxing system; the reflux column was made to project out vertically from the top of the oven. Solutions were refluxed for $30 \mathrm{~min}$ in each case with the power level held at $20 \%$ $(160 \mathrm{~W})$. Sometimes it was necessary to lower or elevate the microwave power level so that the contents did not boil too violently. The contents were allowed to cool, transferred to centrifugation tubes, centrifuged for $30 \mathrm{~min}$ and the fine deposits at the bottom of the tubes were recovered after decanting the supernatant liquid. The deposits were repeatedly washed with acetone and recentrifuged wherever necessary. The wet samples were then dried before further experiments. Decomposition of the metal organics and the acetates in table 1 leads to formation of the respective oxides. The organic products of decomposition are ethers and esters, which escape or remain partly dissolved. It may be noted that the dielectric constant of the solvent in each case is significantly lower than that of the precursor (solute).

The dry samples were heated in a Thermolyne furnace to rid the particles of the capping agent and to crystallize the amorphous products. The temperatures and the soaking times are given in table 2. The X-ray diffraction (XRD) patterns of the samples were examined at several temperatures on a Siemens Diffrac Puls D5005 diffractometer. A typical evolution of the XRD patterns with heat treatment is shown in figure 1 in the case of $\mathrm{Al}_{2} \mathrm{O}_{3}$. The phase identification was done by comparing with published data. In all the cases the as-obtained powders were amorphous and most of them exhibited crystalline patterns when heated to $200-400^{\circ} \mathrm{C}$ (table 2). The amorphous samples heated to $400^{\circ} \mathrm{C}$ or higher were free from residual organics and the capping agent. This was confirmed using infrared spectra (obtained from the Perkin Elmer FT

Table 1. Precursor and solvent materials used in the present study.

\begin{tabular}{lll}
\hline Nano material & \multicolumn{1}{c}{ Precursor material } & \multicolumn{1}{c}{ Solvent } \\
\hline $\mathrm{MgO}$ & Magnesium ethylate (49) & Ethyl acetate (6) \\
$\mathrm{ZnO}$ & Zinc acetate tetrahydrate (41) & Ethanol (24.3) \\
$\mathrm{NiO}$ & Nickel acetate tetrahydrate (37) & Ethanol (24.3) \\
$\mathrm{Al}_{2} \mathrm{O}_{3}$ & Aluminium-tri-sec butoxide (89) & Ethyl acetate (6) \\
$\mathrm{Fe}_{2} \mathrm{O}_{3}$ & Ferric acetate (63) & Ethanol (24.3) \\
$\mathrm{ZrO}_{2}$ & Zirconium (IV) isopropoxide (162) & Ethyl acetate (6) \\
\hline
\end{tabular}


Table 2. Soaking time and sizes of the oxide particles*.

\begin{tabular}{lcccccccc}
\hline & \multicolumn{7}{c}{ Temperature $\left({ }^{\circ} \mathrm{C}\right)$} \\
\cline { 2 - 8 } Material & 200 & 300 & 400 & 600 & 700 & 800 & 1000 & 1200 \\
\hline $\mathrm{MgO}$ & $6 \mathrm{~h}$ & - & $6 \mathrm{~h}$ & $6 \mathrm{~h}$ & - & $6 \mathrm{~h}$ & $1 \mathrm{~h}$ & $6 \mathrm{~h}$ \\
& $\mathrm{a}$ & & $9 \AA$ & $14 \AA$ & & $27 \AA$ & $29 \AA$ & $37 \AA$ \\
$\mathrm{ZnO}$ & $6 \mathrm{~h}$ & $6 \mathrm{~h}$ & - & - & $6 \mathrm{~h}$ & - & $6 \mathrm{~h}$ & - \\
& $16 \AA$ & $17 \AA$ & & & $34 \AA$ & & $41 \AA$ & \\
$\mathrm{NiO}$ & $6 \mathrm{~h}$ & $6 \mathrm{~h}$ & - & - & - & - & - & - \\
& $\mathrm{a}$ & $14 \AA$ & & & & & & \\
$\mathrm{Al}_{2} \mathrm{O}_{3}$ & $6 \mathrm{~h}$ & - & $6 \mathrm{~h}$ & $6 \mathrm{~h}$ & - & $6 \mathrm{~h}$ & $1 \mathrm{~h}$ & $2 \mathrm{~h}$ \\
& $\mathrm{a}$ & & $\mathrm{a}$ & $\mathrm{a}$ & & $7 \AA$ & $9 \AA$ & $32 \AA$ \\
$\mathrm{Fe}_{2} \mathrm{O}_{3}$ & $6 \mathrm{~h}$ & - & $6 \mathrm{~h}$ & $6 \mathrm{~h}$ & - & - & - & - \\
& $\mathrm{a}$ & & $18 \AA$ & $32 \AA$ & & & & \\
$\mathrm{ZrO}_{2}$ & $6 \mathrm{~h}$ & - & $6 \mathrm{~h}$ & $6 \mathrm{~h}$ & - & $6 \mathrm{~h}$ & $1 \mathrm{~h}$ & $2 \mathrm{~h}$ \\
& $\mathrm{a}$ & & $14 \AA$ & $16 \AA$ & & $16 \AA$ & $28 \AA$ & $30 \AA$ \\
\hline
\end{tabular}

*Particle sizes are not given when the phases are amorphous (indicated as ' $a$ ').

Spectrum 1000) as shown in figure 2 for the typical case of zirconia in which the absorption in the region of $\sim 1500 \mathrm{~cm}^{-1}$ due to propyl oxy groups disappeared. Particle sizes were determined using Scherrer formula,

$$
D=k \lambda / \beta \cos \theta
$$

where $D$ is the mean diameter of the grains, $k(=0.96)$ is size factor, $\beta$ the full width at half maximum, $\lambda$ the wave length of the X-rays used and $2 \theta$ the angle at which the

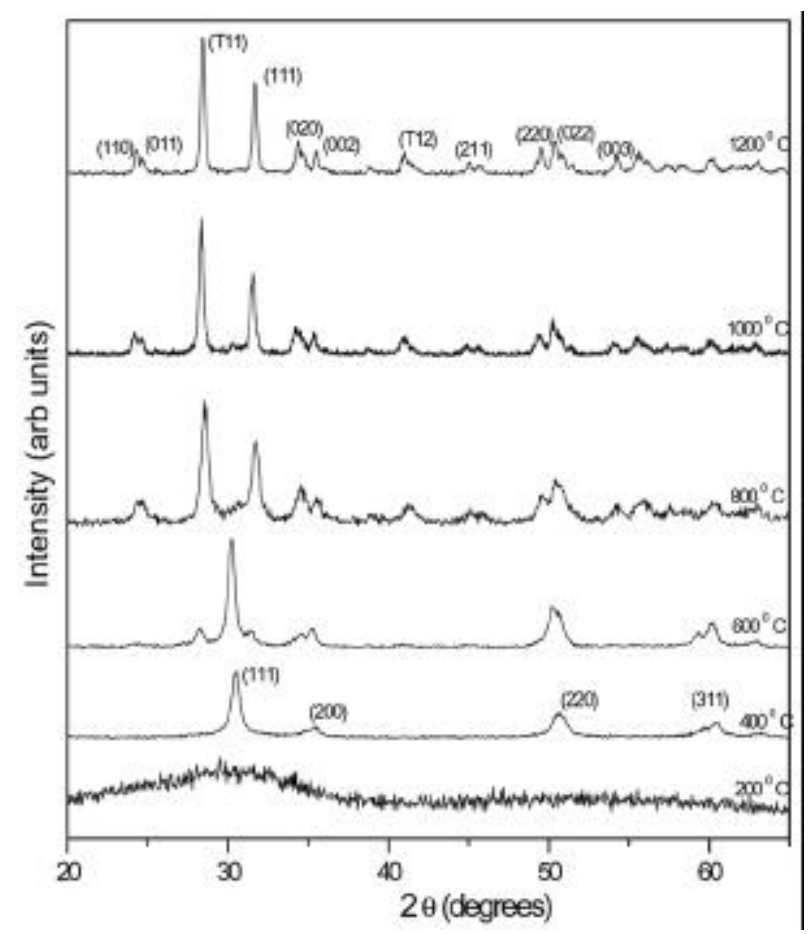

Figure 1. Evolution of the $\mathrm{X}$-ray diffraction pattern with heat treatment at elevated temperatures for the case of $\mathrm{Al}_{2} \mathrm{O}_{3}$.

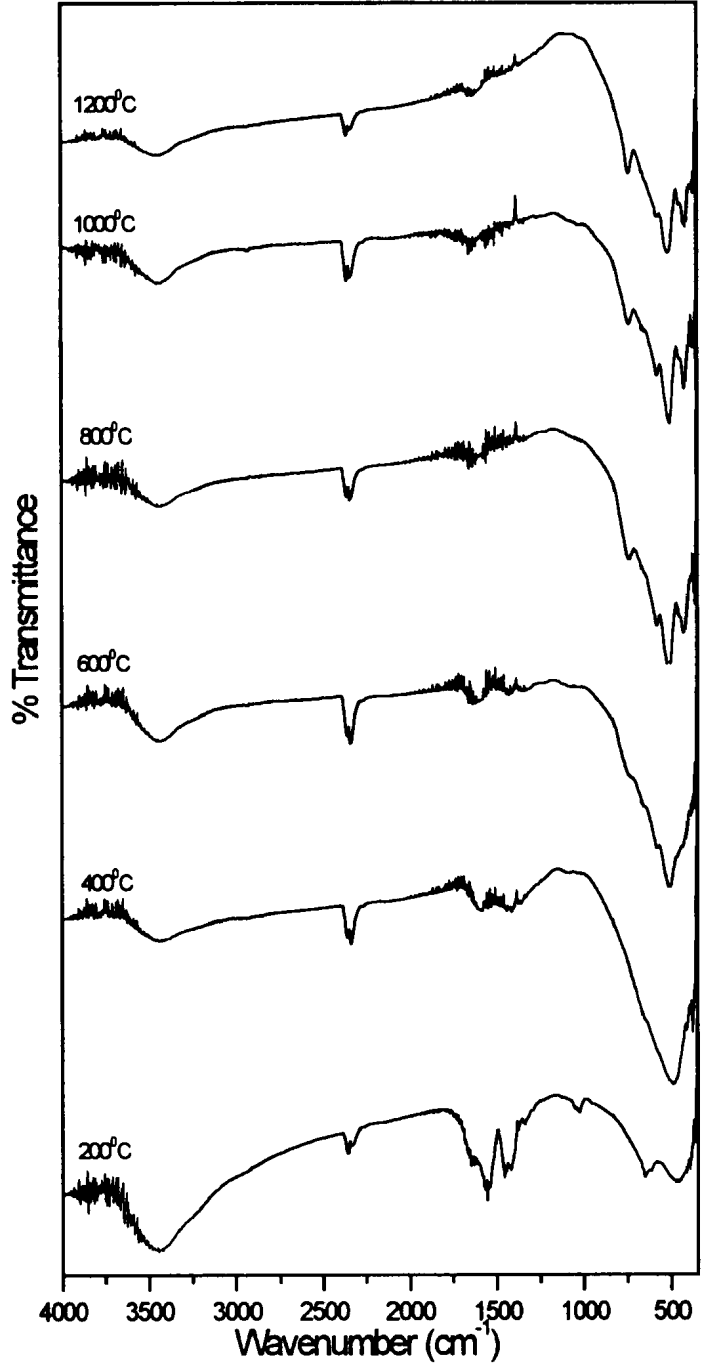

Figure 2. Infrared spectroscopy of the zirconia samples heated to various temperatures. 
maximum intensity was observed. Wherever possible two or three diffraction peaks were chosen and consistency in the particle sizes obtained from using their widths was confirmed. The spread was always within $\pm 2.5 \%$.

\section{Results and discussion}

Particle sizes determined in this manner are given in table 2 for samples heated to various temperatures. Particle sizes were also determined using light scattering methods, by dispersing the samples in ethyl acetate. Particle size distributions for all the samples are given in figure 3 and the thermal histories are indicated in the figure itself.
The agreement between particle sizes obtained from XRD and the sizes indicated by the highest frequency peaks in distribution from the light scattering experiment appear to be quite satisfactory. The particle size distributions are significantly narrow in the case of $\mathrm{ZrO}_{2}$ and $\mathrm{Fe}_{2} \mathrm{O}_{3}$. Particles of $\mathrm{Al}_{2} \mathrm{O}_{3}, \mathrm{ZrO}_{2}$, and $\mathrm{MgO}$ were also examined using transmission electron microscopy (TEM) and the TEM micrographs are shown together in figure 4. Although the particles agglomerate, they are distinguishable and the sizes can be measured. Particle sizes observed in microscopy may be seen to be consistent with the sizes obtained from XRD (table 2) and light scattering measurements.
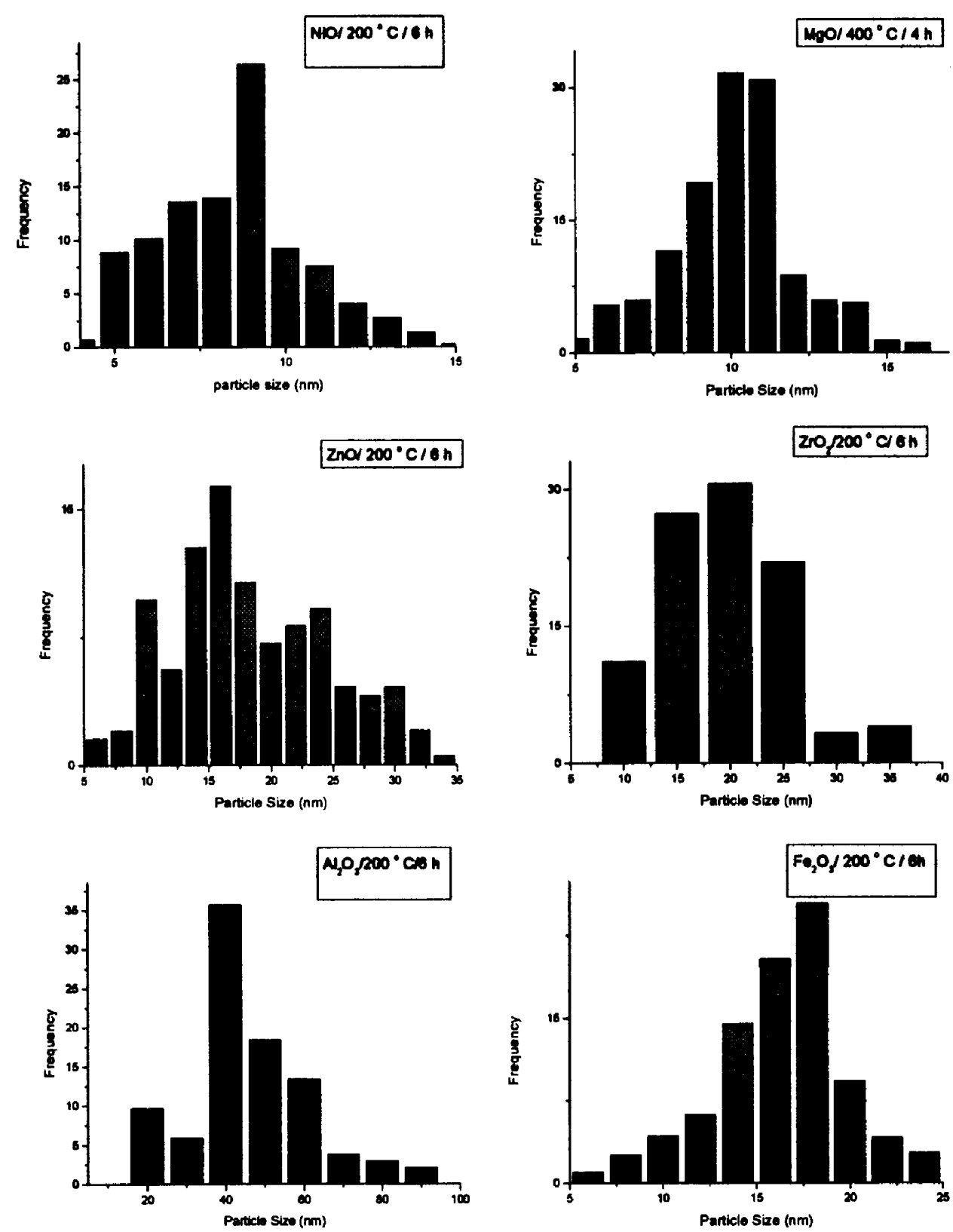

Figure 3. The particle size distributions obtained from the dynamic light scattering experiment. 

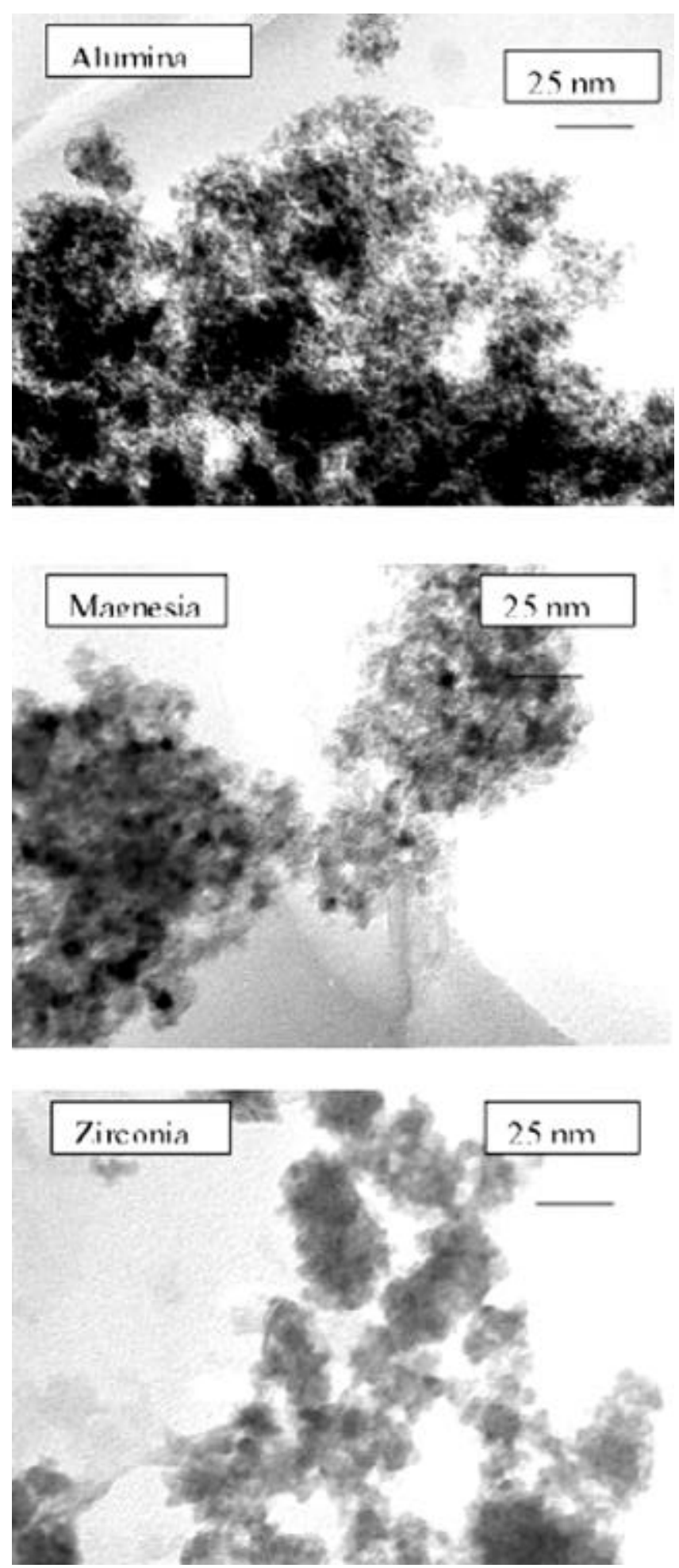

Figure 4. Transmission electron micrographs.

Therefore, in the present microwave approach, nano particles are prepared in a novel and efficient manner as the approach is based on a sound strategy. The primary requirement is that the oxide precursor has to have a dielectric constant, significantly higher than the solvent.
Ethanol is a good solvent in which most of the metal organics are soluble in the range of $5 \%$ by volume. Presence of a suitable capping agent ensures the formation of nanoparticles, which can be later, decomposed. In cases like $\mathrm{ZrO}_{2}$ and $\mathrm{Al}_{2} \mathrm{O}_{3}$, we found formation of nanoparticles even without the use of capping agent. Post preparative heat treatment generally leads to increased nanoparticle sizes. Thus, heat treatment also provides control on the particle sizes of the resulting oxide nanomaterials. The method can be used to prepare other classes of compounds like nitrides and chalcogenides using suitable complexes dissolved in amines and mercaptans, respectively.

\section{Conclusions}

A new strategy of microwave assisted solvothermal method has been applied for the preparation of nanooxides, $\mathrm{MgO}, \mathrm{NiO}, \mathrm{ZnO}, \mathrm{Al}_{2} \mathrm{O}_{3}, \mathrm{Fe}_{2} \mathrm{O}_{3}$ and $\mathrm{ZrO}_{2}$. Based on this new strategy, other nano materials can also be prepared using suitable starting metal organics and capping agents.

\section{Acknowledgement}

The authors acknowledge the financial support provided by the Indian Academy of Sciences, Bangalore.

\section{References}

Agrafiotis C, Tsetsekou A, Stournaras C J, Julbe A, Dalmazio L and Guizard C 2000 Solid State Ionics 1361301

Anderson Kris, Fernández Silvia Cortiñas, Hardacre Christopher and Marr Patricia C 2004 Inorg. Chem. Commun. 773

Ding Tao, Zhang Jian-Rong, Long Su and Zhu Jun-Jie 2003 Microelectronic Eng. 6646

Edelstein A S and Cammarata R C 1998 Nanomaterials: Synthesis, properties and application (Washington DC: Naval Research Laboratory)

Esaki Leo 1999 Nanostructured Mater. 121

He Rong, Qian Xue-feng, Yin Jie, Xi Hong-an, Bian Li-juan and Zhu Zi-kang 2003 Colloids and Surfaces A: Phys. Eng. Aspects 220151

Kaskel Stefan, Schlichte Klaus and Kratzke Tobias 2004 J. Mol. Catal. A: Chem. 208291

Komarneni S 2003 Curr. Sci. 8530

Lee D W and Kim B K 2004 Mater. Lett. 58378

Lee Jin-Seok and Choi Sung-Churl 2004 Mater. Lett. 58390

Pang Qi, Shi Jianxin, Liu Yu, Xing Desong, Gong Menglian and Xu Ningsheng 2003 Mater. Sci. Eng. B103 57

Panneerselvam M 2002 Microwave synthesis and consolidation of advanced ceramics and ceramic composites, $\mathrm{PhD}$ thesis, Indian Institute of Science, Bangalore

Piticescu R R, Barbara Malic, Marija Kosec, Motoc A, Monty C, Iulia Soare, Kosmac T and Daskobler A 2004 J. Euro. Ceram. Soc. 241941

Rao K J, Vaidhyanathan B, Ganguli M and Ramakrishnan P A 1998 Chem. Mater. 88211 
Sankaranarayanan V K and Sreekumar C 2003 Appl. Phys. 3205 Savchuk A I, Paranchych S, Yu, Kurganetski M V, Stolyarchuk I D, Tanasyuk Yu, Perrone V A and DeGiorgi M L 2003 Mater. Sci. Eng. C23 259
Shen Cheng-min, Zhang Xiao-gang, Zhou Ying-ke and Li Hu-lin 2003 Mater. Chem. Phys. 78437

Yin Hengbo, Yamamoto Tetsushi, Wada Yuji and Yanagida Shozo 2004 Mater. Chem. Phys. 8366 